\title{
Retracted: Evaluation by an Aeronautic Dentist on the Adverse Effects of a Six-Week Period of Microgravity on the Oral Cavity
}

\author{
International Journal of Dentistry
}

Received 8 September 2020; Accepted 8 September 2020; Published 3 June 2021

Copyright (c) 2021 International Journal of Dentistry. This is an open access article distributed under the Creative Commons Attribution License, which permits unrestricted use, distribution, and reproduction in any medium, provided the original work is properly cited.

International Journal of Dentistry has retracted the article titled "Evaluation by an Aeronautic Dentist on the Adverse Effects of a Six-Week Period of Microgravity on the Oral Cavity" [1] due to redundant publication with an article published earlier by two of the authors in 2009 [2] and other concerns with the ethics and reporting, as initially raised by Dr. Elisabeth Bik [3, 4]. Dr. Rai does not agree with retraction. Drs. Kaur and Foing did not respond.

\section{Redundant Publication and Experimental Details}

A 2009 article by the same group [2] should have been cited and discussed. The baseline results in Table 2 are identical, and the results in Table 1 are highly similar and some are identical: the baseline amylase means and the $\mathrm{Cl}$ and malonaldehyde means for during simulated microgravity vs. last day of HDT. The ranges/variances are the same at baseline for flow rate, $\mathrm{Na}, \mathrm{K}$, calcium, phosphate, protein, $\mathrm{Cl}$, protein output, amylase, vitamin $\mathrm{E}$, vitamin $\mathrm{C}$, lactate dehydrogenase, MIP 1 alpha, malonaldehyde, 8-hydroxydeoxyguanosine, and thiocynate, and the ranges/variances are the same during simulated microgravity vs. last day of HDT for flow rate, $\mathrm{Na}, \mathrm{K}$, calcium, phosphate, protein, $\mathrm{Cl}$, protein output, amylase, vitamin $\mathrm{E}$, vitamin $\mathrm{C}$, lactate dehydrogenase, MIP 1 alpha, malonaldehyde, 8-hydroxydeoxyguanosine, and thiocynate.

Dr. Rai said the studies had many of the same participants, i.e., volunteers who were members of the Joint Board of Research (JBR Institute of Health Education Research \& Technology, India, the first affiliation of Dr. Rai), who were given the same diet and supplements. However, the age ranges do not overlap: 20 participants aged 18-22 in 2009 (though the abstract says 40 participants) versus 10 participants aged $22-30$ in 2011 . No information about a standard diet and supplements was included in the article or in the consent form and study protocol provided by Dr. Rai.

The exact protocol used in the study is unclear; the study protocol provided by Dr. Rai is brief and lacks additional detail compared to the article. The article implied that participants stayed in the head-down-tilt position for 8 hours, but Dr. Rai clarified that the participants were allowed to leave the position to go to the toilet. However, Dr. Bik noted that, in another study by the same group also published in 2011 [5], it was stated that participants stayed in the head-down-tilt position for 60 days, even during meals, showers, and toilet visits, and compliance was monitored by video. These protocols are therefore inconsistent.

There are further inconsistencies and missing information:

(i) Details of the protocol are missing from the 2011 article [1] compared to the 2009 article [2]. For example, the earlier article says the participants had eight hours head-down-tilt and then four hours recovery in a chair.

(ii) The consent form says "about 20 people" would be recruited, whereas the study protocol says "ten to thirty healthy controls."

(iii) The protocol mentions "controls" rather than "participants."

(iv) The protocol discusses measuring heart rate variability during sleep, but sleep studies are not mentioned in the consent form or article. 
(v) It is not stated when the study began and ended, what the roles of the authors were in the research, and whether anyone other than the authors contributed to the study.

(vi) It is not clear whether the differences reported in Tables 1 and 2 were statistically significant nor whether the numbers in brackets in Table 1 are ranges or variances (e.g., 95\% confidence intervals, standard error, or standard deviation).

Dr. Rai did not provide anonymised individual data points at all timepoints for all outcomes and measurements when this was requested.

\section{Ethics}

Dr. Rai provided a blank copy of the written informed consent document, which only briefly refers to what could be serious adverse effects (e.g., infection) without describing the likelihood or medical monitoring procedures. The consent form only refers to "the influence of isolated environment on human physiology and psychology," not that participants would be subject to six weeks of head-down-tilt bed rest. The number is "IRB \#2013," which appears to correspond to a study done in 2013, not 2011.

The ethical approval document is signed by Prof. Suresh C. Anand, who has been a collaborator of Dr. Rai since 2006, when they were affiliated to the Government Dental College, and they were collaborators at the time of the study: this is not evidence of independent ethical approval. Because Prof. Anand was actively collaborating with Drs. Rai and Kaur and he was listed as the patron of the JBR Health Education and Research Organization, he should have recused himself from any ethical approval process at JBR.

The institutional review board (IRB) approval was given on January 5, 2010, but the study protocol cites two publications from 2010 (Rai et al. 2010 and Vetter et al. 2010); because the study protocol should have been approved by the IRB and then not altered, this is concerning. It is unclear which article is meant by Vetter et al., 2010, because there is no published fatigue scale by anyone by that name.

Dr. Rai said the participants were members of the Joint Board of Research, which was not disclosed in the article. Because Dr. Rai found and is president of this organisation, the use of these participants created a risk of coercion because they may have felt obliged to volunteer for this research. The 2006 Indian Council of Medical Research (ICMR) guidelines that applied to this research state "adequate justification is required for the involvement of participants such as prisoners, students, subordinates, employees, service personnel etc. who have reduced autonomy as research participants, since the consent provided may be under duress or various other compelling reasons" [6]. No such justification was provided. This is particularly relevant in the absence of independent ethics oversight.
The consent form says that no compensation would be paid in the event of injury. However, the ICMR guidelines [6] state "Research participants who suffer physical injury as a result of their participation are entitled to financial or other assistance to compensate them equitably for any temporary or permanent impairment or disability."

\section{Text Similarity}

There is text overlap with four uncited articles [7-10], which should have been cited at their point of use. Their wording should not have been used without explicit quotation:

(i) 130 words from Heer, M., Boerger, A., Kamps, N. et al., "Nutrient supply during recent European missions," Pflügers Arch - Eur J Physiol (2000) 441 (Suppl 1): R8. https://doi.org/10.1007/s004240000334

(ii) 50 words from Stuempfle, K., and D. Drury. The Physiological Consequences of Bed Rest. Journal of Exercise Physiology online (June 2007) 10 (3): 32-41.

(iii) 30 words from Olabi, A., Lawless, H., Hunter, J., Levitsky, D. and Halpern, B. (2002), The Effect of Microgravity and Space Flight on the Chemical Senses. Journal of Food Science, 67: 468-478. doi:10.1111/j. 1365-2621.2002.tb10622.x https://onlinelibrary.wiley. com/doi/abs/10.1111/j.1365-2621.2002.tb10622.x

(iv) 30 words from Elisabeth Blaber, Helder Marçal, and Brendan P. Burns, "Bioastronautics: The Influence of Microgravity on Astronaut Health," Astrobiology 2010 10: 5, 463-473 https://www.liebertpub.com/ doi/abs/10.1089/ast.2009.0415

\section{Affiliations}

The affiliations of Balwant Rai to the Kepler Space Institute and Jasdeep Kaur to KU Leuven (Catholic University, Leuven) are disputed by these institutions and should not have been used.

\section{References}

[1] B. Rai, J. Kaur, and B. H. Foing, "Evaluation by an Aeronautic Dentist on the Adverse Effects of a Six-Week Period of Microgravity on the Oral Cavity," International Journal of Dentistry, vol. 2011, Article ID 548068, 5 pages, 2011.

[2] B. Rai and J. Kaur, "Effect of Microgravity on Oral Cavity: Mission to Mars," Indian Journal of Dental Education, vol. 2, no. 1, 2009.

[3] E. Bik, "The Space Dentist," Science Integrity Digest, 2019.

[4] E. Bik, "Evaluation by an Aeronautic Dentist on the Adverse Effects of a Six- Week Period of Microgravity on the Oral Cavity," PubPeer, 2019.

[5] B. Rai, J. Kaur, M. Catalina, S. C. Anand, R. Jacobs, and W. Teughels, "Effect of Simulated Microgravity on Salivary and Serum Oxidants, Antioxidants, and Periodontal Status. 
Journal of Periodontology," Journal of Periodontology, vol. 82, no. 10, pp. 1478-1482, 2011, doi: 10.1902/jop.2011.100711.

[6] "Ethical Guidelines for Biomedical Research on Human Participants," Indian Council of Medical Research, New Delhi, 2006, https://ethics.ncdirindia.org//asset/pdf/ICMR_ethical_guidelines_ for_biomedical_research_for_human_participants_2006.pdf.

[7] M. Heer, A. Boerger, N. Kamps, C. Mika, C. Korr, and C. Drummer, "Nutrient supply during recent European missions," Pflügers Archiv, vol. 441, no. 1, p. R8, 2000, doi: $10.1007 / \mathrm{s} 004240000334$.

[8] K. Stuempfle and D. Drury, "The Physiological Consequences of Bed Rest," Journal of Exercise Physiology Online, vol. 10, no. 3, pp. 32-41, 2007.

[9] A. A. Olabi, H. T. Lawless, J. B. Hunter, D. A. Levitsky, and B. P. Halpern, "The Effect of Microgravity and Space Flight on the Chemical Senses. Journal of Food Science," Journal of Food Science, vol. 67, no. 2, pp. 468-478, 2002, doi: 10.1111/ j.1365-2621.2002.tb10622.x.

[10] E. Blaber, H. Marçal, and B. P. Burns, "Bioastronautics: The Influence of Microgravity on Astronaut Health," Astrobiology, vol. 10 , no. 5, pp. 463-473, 2010, doi: 10.1089/ast.2009.0415. 


\title{
Evaluation by an Aeronautic Dentist on the Adverse Effects of a Six-Week Period of Microgravity on the Oral Cavity
}

\author{
Balwant Rai, 1, 2,3 Jasdeep Kaur, ${ }^{1,4}$ and Bernard H. Foing ${ }^{3}$ \\ ${ }^{1}$ JBR Institute of Health Education Research \& Technology, India \\ ${ }^{2}$ Kepler Space University, Bluffton, SC 29909, USA \\ ${ }^{3}$ Faculty of Earth \& Life Sciences, Vrije Universiteit Amsterdam \& ILEWG, Amesterdam, The Netherlands \\ ${ }^{4}$ Catholic University, Leuven, Belgium
}

Correspondence should be addressed to Balwant Rai, drbalwantraissct@rediffmail.com

Received 2 July 2011; Revised 7 October 2011; Accepted 8 October 2011

Academic Editor: Francesco Carinci

Copyright ( $) 2011$ Balwant Rai et al. This is an open access article distributed under the Creative Commons Attribution License, which permits unrestricted use, distribution, and reproduction in any medium, provided the original work is properly cited.

Objective. HDT bed rest condition is a simulated microgravity condition in which subject lies on bed inclined -6 degree feet up. To determine the influence of a simulated microgravity (HDT bed rest) on oral cavity, 10 healthy male volunteers were studied before, during, just after, and after 6 weeks of the simulated microgravity condition of $-6^{\circ}$ head-down-tilt (HDT) bed rest. Materials and Methods. Facial nerve function, facial sensation, chemosensory system, salivary biomarkers were measured. Results. Lactate dehydrogenase, MIP 1 alpha, malonaldehyde, 8-hydroxydeoxyguanosine, and thiocyanate were found to increase significantly, while flow rate, sodium, potassium, calcium, phosphate, protein, amylase activity, vitamin E and C, and mouth opening were decreased in simulation environments in contradiction to normal. The threshold for monosodium glutamate (MSG) and capsaicin increased during microgravity as compared to normal conditions. Moderate pain of teeth, facial oedema, mild pain, loss of sensation of pain and temperature, decreased tongue, and mandibular movement in simulation microgravity environments were observed. Conclusions. These results suggest that reversible effect of microgravity is oedema of face, change in taste, abnormal expression of face, teeth pain, and xerostomia. Further study will be required on large scale on long-term effects of microgravity on oral cavity to prevent the adverse effects.

\section{Introduction}

For many years, the prevailing concept in space human factors research has been that microgravity has an impact on human physiology and astronauts are faced with several health risks during both short- and long-duration spaceflights. Some of these health problems include bone loss, muscle atrophy, cardiac dysrhythmias, and altered orientation $[1,2]$. It has been reported that saliva composition is changed and oral health compromised during simulated skylab mission [3,4]. Previous studies observed some adverse effects of simulated microgravity (HDT bed rest condition) on oral cavity [5-12]. HDT bed rest condition is a simulated microgravity condition in which subject lies on bed inclined -6 degree feet up [2].This study is extension of our previous studies [6]. To the best of our knowledge, no long-term study has been conducted on effect of oral cavity such as facial nerve functions, prevalence and pattern of oral disease, bone loss, tooth pain, salivary flow rate, and different salivary biomarkers. The prevalence of oral diseases such as dental caries, periodontal disease and cancer, and bone loss was estimated by salivary biomarkers, which is noninvasive. Hence, this study was planned to evaluate the effect of simulated microgravity on oral cavity.

\section{Materials and Methods}

The subjects of this investigation were 10 male volunteers (aged 22-30 years, body mass index 18.7 (2.6), participated in a 6-week $6^{\circ}$ HDT bed-rest position). We chose 6-week duration of study taken into account for three continues ISS mission for astronaut. Females were not selected in this study because females were not willing to take part in this study. 
Each subject was given a detailed explanation of the experimental protocol and provided written and verbal consent. The average energy, calcium, and vitamin $\mathrm{D}$ expended by the subjects during the simulation was $2300 \mathrm{kcal} /$ day (range 2080-3010 kcal/day), $1200 \mathrm{mg} /$ day, and $10 \mu \mathrm{g}$, respectively. Each subject completed a questionnaire on their medical and dental history to determine the status of systemic diseases, smoking, and history of alcohol and drug use. They also underwent a clinical examination for systemic diseases, chronic diseases such as autoimmune diseases, cancer, cardiovascular diseases such as cerebrovascular diseases, heart failure, ischemic cardiopathy, chronic fatigue syndrome, chronic graft-versus-host disease (GVHD), chronic hepatitis, chronic pain syndromes, chronic osteoarticular diseases, chronic renal failure, chronic respiratory diseases, and diabetes mellitus, and oral and dental diseases. Patients were excluded if they had a systemic or chronic disease, an oral or dental disease, if they were smokers, or if they had a history of alcohol or drug abuse. Subjects with caries, cavities, sealants, nontreated cavities, mal-adjusted crowns, and previous channel treatments were excluded. All parameters and samples were taken just before HDT and last day of HDT. Facial function tests, mouth opening, jaw movements, tongue movements, facial sensation (touch, pressure, temperature), taste, odor, perception of food, salivary vitamins E and C, lactate dehygrogenase isoenzyme, MIP 1 alpha, glucosyltransfer B, malonaldehyde, 8-hydroxydeoxyguanosine, thiocyanate, salivary contents, and salivary flow rate were measured as in our previous studies [5-12]. To measure pain, we used a visual scale analog ranging from 0.5 (very mild pain) to 5 (severe pain). Change in mouth opening was measured. Salivary vitamin $\mathrm{C}$ and vitamin E were estimated by HPLC. HPLC separations were accomplished at room temperature (approximately $370^{\circ} \mathrm{C}$ ) with a cecil liquid chromatography system (series, 1100 , USA) consisting of a sample injection valve with a $30 \mathrm{u}$ and sample loop, an ultraviolet ( $\mathrm{mv}$ ) spectrophotometric detector, a integrator, and techsphere ODS-2 packed (4 um particle and 80.4 pore size $)$ column $(250 \times 4.6$ ID $)$ with a methanol: acetonitrile: chloroform $(45: 41: 10 \mathrm{~V} / \mathrm{V})$ as mobile phase at $1 \mathrm{~mL} / \mathrm{min}$ flow rate. All procedures were performed under light protected conditions $[6,13]$. MIP 1 alpha, lactate dehygrogenase isoenzyme, and amylase levels were measured by using ELISA kit (MIP 1 alpha, R\&D Systems, Inc._Minn, USA; L type LDH J; Wako Chemical Industry, Osaka, Japan; Yanaihara Institute, Shizuoka, Japan). We determined glucosyltransfer B present in the salivary samples using an ELISA assay by employing a kit assay obtained from Kirkegaard and Perry Laboratories, Gaithersburg, Md, USA [6]. Salivary 8OHdG levels were measured by a competitive ELISA kit (Japan Institute for control of Aging, Shizuoka, Japan) [6]. Lipid peroxidation product MDA was analyzed by thiobarbituric acid (TBA) reaction $[6,14]$. Saliva was assessed colorimetrically by a spectrophotometer and using affiliated kits for analysis of saliva sodium, potassium, calcium, and phosphate. The total protein concentration was measured by the biuret method [6]. Salivary 8-OHdG levels were measured by using (ELISA kit-competitive method, Cayman Chemical, USA). The data were analyzed using SPSS version 11 and applied the student $t$-test.

\section{Results}

Lactate dehygrogenase, MIP 1 alpha, malonaldehyde, 8-hydroxydeoxyguanosine, and thiocyanate were significantly higher; while flow rate, sodium, potassium, calcium, phosphate, protein, amylase activity, and vitamins $\mathrm{E}$ and $\mathrm{C}$ were decreased in HDT as compared to normal (Table $1, P<$ 0.05). The threshold for MSG and capsaicin was increased about 1.5 dilution step, while sodium chloride was decreased about 2 dilution during microgravity as compared to normal (Table 2). Pain scores were increased in teeth, mandibular angle regions, sublingual and submandibular opening duct regions HDT as compared to normal (Table 3 ). Face, submandibular and sublingual duct opening area showed swelling in HDT as compared to normal (Table 3). Face showed abnormal expression during HDT. Mouth opening, tongue, and mandibular movement were decreased in HDT as compared to normal, although levels were not statically significant (Tables 2 and 3).

\section{Discussion}

Flow rate, sodium, potassium, calcium, phosphate, and protein levels were increased in simulation environments as compared to normal, while same findings were observed in urine $[6,15]$. Increased bone resorptions contribute significantly to raise the salivary state of saturation with respect to the calcium salts, namely, calcium oxalate and calcium phosphate [6]. In addition, other environmental and dietary factors may adversely affect salivary composition and increase stone formation risk during space flight $[15,16]$. Although observations to date have suggested that there could actually be a reduced food intake during the early phase of flight, crew members on longer-duration flights could also increase food intake and be at increased risk for salivary stone formation $[6,17]$. The most important effect of restricting energy intake is on calcium and bone metabolism. The MIP 1 alpha level was decreased in microgravity which is potential markers of bone loss $[9,16]$. In agreement with earlier reports by Parazynski et al. [17], an increased fluid excretion was observed in simulated microgravity, which leads to dehydration and finally to a reduction of plasma volume and an increase in the hematocrit. A reduction of plasma volume may result in increase in serum electrolyte levels, and therefore serum osmolality and urine osmolality increase too. The plasma volume decreases together with increases in serum and urine osmolality and electrolyte levels, influences body fluid regulation by activating hormonal regulatory factors, that is, vasopressin, rennin, and aldosterone [17]. In agreement with earlier reports by Kirsch et al. [18], plasma volume contraction occurs quickly in microgravity. This hemoconcentration probably results from increased upperbody vascular pressures in microgravity [16] and perhaps reduced interstitial pressures; both factors would encourage transcapillary fluid filtration into upperbody interstitial spaces, and substantial filtration can occur in minutes [19].

The levels of calcium were increased in microgravity as compared to control as reported in our previous study [6]. MIP 1 alpha was elevated during HDT condition as 
TABle 1: The median (range) unstimulated salivary whole flow rate, compositions, biomarkers (sodium, potassium, calcium, phosphate, protein, lactate dehygrogenase, MIP 1 alpha, malonaldehyde, 8-hydroxydeoxyguanosine, thiocyanate, amylase activity, vitamins E, C), and mouth opening before and during last day of HDT in 10 healthy persons.

\begin{tabular}{|c|c|c|c|}
\hline Parameters & Before HDT & Last day of HDT & $P$ value \\
\hline Flow rate $(\mathrm{mL} / \mathrm{min})$ & $0.026(0.01-0.03)$ & $0.009(0.008-0.02)$ & $<0.01$ \\
\hline $\mathrm{Na}(\mathrm{mM})$ & $13(8.0-15.0)$ & $12(9.0-18.2)$ & $<0.01$ \\
\hline $\mathrm{K}(\mathrm{mM})$ & $23.6(6.7-34.8)$ & $22.2(7.3-36.9)$ & $<0.05$ \\
\hline Total calcium (mM) & $3.4(1.4-4.6)$ & $3.9(2.4-4.8)$ & $<0.01$ \\
\hline Total phosphate (mM) & $7.4(1.3-11.3)$ & $7.8(2.1-12.1)$ & $<0.01$ \\
\hline Total protein $(\mathrm{mg} / \mathrm{mL})$ & $4.4(1.6-13.6)$ & $4.5(1.7-14.2)$ & $<0.05$ \\
\hline $\mathrm{Cl}(\mathrm{Mm})$ & $22.3(14.9-42.1)$ & $22.0(14.7-43.2)$ & $<0.05$ \\
\hline Total protein output (mg/min) & $0.44(0.23-0.96)$ & $0.42(0.26-0.85)$ & $<0.001$ \\
\hline Amylase activity (micro kat/L) & $324(145-567)$ & $262(112-345)$ & $<0.001$ \\
\hline Vitamins E (mg/mL) & $0.56(0.32-0.76)$ & $0.51(0.31-0.73)$ & $<0.005$ \\
\hline Vitamins C (mg/mL) & $0.35(0.12-54)$ & $0.32(0.09-0.52)$ & $<0.05$ \\
\hline Lactate dehygrogenase (IU/L) & $2.4(1.2-3.4)$ & $2.3(1.6-3.7)$ & $<0.05$ \\
\hline MIP 1 alpha (pg/mL) & $17.6(12.6-21.5)$ & $18.1(13.2-22.4)$ & $<0.01$ \\
\hline Malonaldehyde (ng/mL) & $2.46(1.45-4.34)$ & $2.65(1.67-4.89)$ & $<0.05$ \\
\hline 8-hydroxydeoxyguanosine (ng/mL) & $0.66(0.45-1.34)$ & $0.78(0.51-1.43)$ & $<0.001$ \\
\hline Thiocyanate (U/L) & $34.3(21.7-56.9)$ & $39.8(22.7-57)$ & $<0.001$ \\
\hline Mouth opening (In cm) & $45.4(34.6-53.2)$ & $42.3(31.5-49.6)$ & $<0.005$ \\
\hline
\end{tabular}

TABLE 2: The square means of the thresholds of all persons before and on last day of HDT in 10 healthy persons. The thresholds are reported as the dilution series values (dilution 10 is most conc., dilution 1 is least conc.).

\begin{tabular}{lccc}
\hline Parameters & $\begin{array}{c}\text { Before } \\
\text { HDT }\end{array}$ & $\begin{array}{c}\text { Last day of } \\
\text { HDT }\end{array}$ & $\begin{array}{c}P \\
\text { value }\end{array}$ \\
\hline Sucrose & 3.8 & 3.3 & $<0.05$ \\
Citric acid & 3.1 & 2.4 & $<0.05$ \\
Sodium chloride & 3.4 & 2.2 & $<0.05$ \\
Quinine & 4.6 & 4.1 & $<0.05$ \\
MSG & 6.2 & 6.9 & $<0.05$ \\
Capsaicin & 6.4 & 7.1 & $<0.05$ \\
Amyl butyrate & 3.2 & 2.8 & $<0.05$ \\
Methone & 3.1 & 2.5 & $<0.05$ \\
\hline & & &
\end{tabular}

biomarker of bone loss as reported in previous studies [6, 16]. Loss of bone calcium during bed rest is the result of increased bone resorption by osteoclasts and it is not due to endocrine changes. Parathyroid hormone (PTH) promotes the release of calcium from bone and stimulates the kidneys to release the active form of Vitamin D, 1,25-dihydroxyvitamin $\mathrm{D}$, which increases intestinal calcium absorption [18-23]. Insufficient calcium consumption leads to a reduction in serum calcium levels and thereby to a secretion of parathyroid hormone (PTH) and calcitriol synthesis. Both rises in PTH and calcitriol induce an increase in calcium retention either from the intestine or from bone. Based on that, a long-lasting insufficient calcium intake and insufficient vitamin $\mathrm{D}$ are the main factors leading to a decrease in bone mineral density $[20,21]$. It might lead to periodontitis and facture of facial bones.

The decreased levels of vitamins $\mathrm{E}$ and $\mathrm{C}$ and increased malonaldehyde levels denoted increase in free radical activity as in microgravity environments [21]. So the free radical activity increased in microgravity as compared to normal gravity as reported in previous studies [21]. The 8-hydroxy deoxyguanosine levels were increased in saliva in microgravity environments as compared to normal, it may be due to increase in oxidative stress $[6,17,21]$. The threshold for MSG and capsaicin increased about 1.5 dilution step, while sodium chloride decreased about 2 dilution during microgravity as compared to normal. It might be due to fluid shift mechanism. It could be due to physiological changes including an upward shift of body fluids toward the head, which may lead to an attenuation of the olfactory component in the flavour of foods, pressing the nerve regions or dysfunction of nerve as well as increased activity of b-AR agonists [21]. Lactate dehygrogenase isoenzyme levels increased during HDT, this implied the development of muscular atrophy as reported in previous studies [23]. Salivary glucosyltransferase B levels were increased in HDT as compared to normal as reported in previous study [6] and indicated that caries prevalence is more during microgravity. Thiocyanate levels were significantly increased in HDT as compared to normal as reported in previous study [6]. Thiocyanates (SCN-) are ubiquitous in nature. There are indispensable parts of host defense system that act as a substrate for lactoperoxidase (LPO). LPO oxidizes saliva SCN-thereby generating antimicrobial agent hypothiocyanite (OSCN-) [6]. Pain scores increased in teeth and mandibular angle regions in HDT as compared to normal. It might be due to the pain mechanism appears somatic due to excessive expansion. Sublingual and 
TABLE 3: The symptoms of subjects before and on last day of HDT in 10 healthy persons.

\begin{tabular}{lcl}
\hline Parameters & Before HDT & Last day of HDT \\
\hline Teeth pain while articulation of teeth & 0 & 1.0 \\
$\begin{array}{l}\text { Facial swelling } \\
\text { Pain while closing or opening the mouth }\end{array}$ & No swelling & Moderated oedema \\
Salivary gland & 0 & 0.5 \\
$\begin{array}{l}\text { Pain while moderate pressing the facial } \\
\text { region }\end{array}$ & Normal & $\begin{array}{l}\text { Moderated pain in submandibular and sublingual duct } \\
\text { opening area and swelling }\end{array}$ \\
$\begin{array}{l}\text { Facial expression } \\
\text { Sensation tests }\end{array}$ & 0.5 \\
$\begin{array}{l}\text { Movements of tongue } \\
\text { Jaw movements }\end{array}$ & Normal & Abnormal \\
\hline
\end{tabular}

submandibular opening duct regions, abnormal facial expression, loss of sensation of pain and temperature, decreased tongue, and mandibular movements in simulation microgravity environment were observed due to fluid shift mechanism.

The limitations of this study are short duration, small sample size, female subjects who were not included, no followup of volunteers, and other factors. These results suggest that reversible effect of microgravity is oedema of face, change in taste, abnormal expression of face, teeth pain, and xerostomia. The nonreversible effects of microgravity such as periodontal diseases and dental caries occur in different pattern than normal, and stone formation in salivary duct, precancer or cancer, fracture of maxillary and mandibular bone, and xerostomia are more prevalent in astronauts as compared to normal persons. Further study will be required on large-scale and on long-term effects of microgravity on oral cavity to prevent the adverse effects.

\section{References}

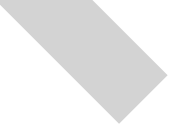

[1] S. Herault, G. Fomina, I. Alferova, A. Kotovskaya, V. Poliakov, and P. Arbeille, "Cardiac, arterial and venous adaptation to weightlessness during 6-month MIR spaceflights with and without thigh cuffs (bracelets)," European Journal of Applied Physiology, vol. 81, no. 5, pp. 384-390, 2000.

[2] V. S. Oganov, A. Grigor'ev, L. I. Voronin et al., "Bone mineral density in cosmonauts after flights lasting 4.5-6 monthson the Mir orbital station ," Aviakosmicheskaia i Ekologicheskaia Meditsina, vol. 26, no. 5-6, pp. 20-24, 1992.

[3] L. R. Brown, W. J. Frome, and M. G. Wheatcroft, "The effect of skylab on the chemical composition of saliva," Journal of Dental Research, vol. 56, no. 10, pp. 1137-1143, 1977.

[4] D. K. Savage, "A brief history of aerospace dentistry," Journal of the History of Dentistry, vol. 50, no. 2, pp. 71-75, 2002.

[5] B. Rai and J. Kaur, "Oral cavity in simulated microgravity: a dentist on Mars," in Proceedings of the 18th IAA Humans in Space Symposium, Houston, Tex, USA, April 2011, http://www .dsls.usra.edu/meetings/IAA/pdf/2002.pdf.

[6] B. Rai, "Human oral cavity in simulated microgravity: new prospects," Advances in Medical and Dental Sciences, vol. 3, no. 1, pp. 35-39, 2009.
[7] B. Rai and J. Kaur, "Salivary stress markers and psychological stress in simulated microgravity: 21 days in 60 head-down tilt," Journal of Oral Science, vol. 53, no. 1, pp. 103-107, 2011.

[8] B. Rai, J. Kaur, M. Catalina, S. C. Anand, R. Jacobs, and W. Teughels, "Effect of simulated microgravity on salivary and serum oxidants, antioxidants, and periodontal status," Journal of Periodontology, vol. 82, no. 10, pp. 1478-1482, 2011.

[9] B. Rai, J. Kaur, and M. Catalina, "Bone mineral density, bone mineral content, gingival crevicular fluid (matrix metalloproteinases, cathepsin $\mathrm{K}$, osteocalcin), and salivary and serum osteocalcin levels in human mandible and alveolar bone under conditions of simulated microgravity," Journal of Oral Science, vol. 52, no. 3, pp. 385-390, 2010.

[10] B. Rai, J. Kaur, and M. Catalina, "Bone mineral density, Bone mineral contents, MMP-8 and MMP-9 levels in Human Mandible and alveolar bone: simulated microgravity," in Proceedings of the 38th COSPAR Scientific Assembly, p. 2, Bremen, Germany, July 2010.

[11] B. Rai and J. Kaur, "Odorant identification based on solid phase in EVA Participants," Special Science report (Biomedical), 2011. http://mdrs.marssociety.org/home/field-reports/ crew100b/day12.

[12] B. Rai and J. Kaur, "The history and importance of aeronautic dentistry," Journal of Oral Science, vol. 53, no. 2, pp. 143-146, 2011.

[13] Y. Nomura, Y. Tamaki, T. Tanaka et al., "Screening of periodontitis with salivary enzyme tests," Journal of Oral Science, vol. 48, no. 4, pp. 177-183, 2006.

[14] J. A. Buege and S. D. Aust, "Microsomal lipid peroxidation," Methods in Enzymology, vol. 52, no. C, pp. 302-310, 1978.

[15] J. E. Zerwekh, "Nutrition and renal stone disease in space," Nutrition, vol. 18, no. 10, pp. 857-863, 2002.

[16] D. H. Fine, K. Markowitz, D. Furgang et al., "Macrophage inflammatory protein-1 $\alpha$ : a salivary biomarker of bone loss in a longitudinal cohort study of children at risk for aggressive periodontal disease?" Journal of Periodontology, vol. 80, no. 1, pp. 106-113, 2009.

[17] S. E. Parazynski, A. R. Hargens, B. Tucker, M. Aratow, J. Styf, and A. Crenshaw, "Transcapillary fluid shifts in tissues of the head and neck during and after simulated microgravity," Journal of Applied Physiology, vol. 71, no. 6, pp. 2469-2475, 1991.

[18] K. A. Kirsch, L. Röcker, O. H. Gauer et al., "Venous pressure in man during weightlessness," Science, vol. 225, no. 4658, pp. 218-219, 1984.

[19] D. E. Watenpaugh and M. L. Smith, "Human cardiovascular acclimation to microgravity," Journal of Gravitational Physiology, vol. 5, no. 1, pp. P15-P18, 1998. 
[20] F. Bronner, "Calcium and osteoporosis," The American Journal of Clinical Nutrition, vol. 60, no. 6, pp. 831-836, 1994.

[21] Z. M. Vickers, B. L. Rice, M. S. Rose, and H. W. Lane, "Simulated microgravity [bed rest] has little influence on taste, odor or trigeminal sensitivity," Journal of Sensory Studies, vol. 16, no. 1, pp. 23-32, 2001.

[22] J. -H. Xue, L. -H. Chen, H. -Z. Zhao et al., "Differential regulation and recovery of intracellular $\mathrm{Ca} 2+$ in cerebral and small mesenteric arterial smooth muscle cells of simulated microgravity rat," PLoS One, vol. 6, no. 5, Article ID e19775, 2011.

[23] H. Saiki, M. Nakaya, M. Sudoh, T. Okamoto, and J. Nakajima, "Changes in enzymes and potassium content of the neuromuscular systems of albino rats during prolonged exposure to simulated hypogravics," Life Sciences and Space Research, vol. 17, pp. 205-211, 1979. 